\title{
Stability Analysis of the Flow of Blood in the Branching and Stenoted Arteries
}

Rotaiyna J. Essa

RotinaJasim@yahoo.com

College of Computer Science and Mathematics

University of Mosul, Mosul, Iraq

Received on: 19/03/2012

Shaimaa M. Younis

Shes7369@gmail.com

\section{ABSTRACT}

This research is devoted to the stability analysis of the system of equations that describes the flow of blood in the branching and stenoted arteries. This branch takes the form of the character $\mathrm{Y}$, By using the Navier -Stoke of equations in Polar coordinates, when the amplitude is constant and we found that the system is stable under the condition $\frac{1}{\operatorname{Re}}<\frac{\frac{1}{r}\left(k_{1}^{2} u_{1}+k_{1} k_{2} w_{1}\right)}{\frac{1}{r^{2}}\left(k_{1}^{2}-r^{2} k_{1}^{2} k_{2}^{2}-k_{2}^{2}-r^{2} k_{2}^{4}\right)}$

Keywords:,stenoted arteries ,flow of blood, stability analysis , Dimensional analysis

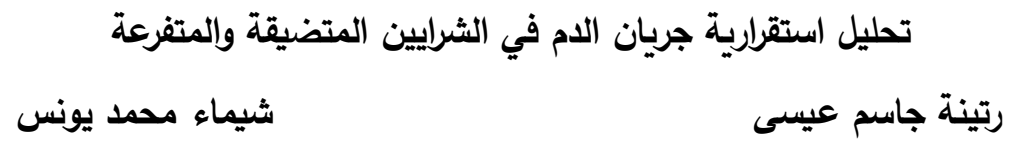

$$
\text { جلية علوم الحاسوب واليراضيات الموصل، الموصل، العرلق }
$$

تاريخ قبول البحث: 2012/06/28

تاريخ استلام البحث: 2012/03/19

\section{الملخص}

هذا البحث مكرس لتحليل الاستقرارية لمنظومة من المعادلات التي تصف تدفق الدم في الشرايين المتقرعة والمتضيقة وهذا التفرع يأخذ شكل الحرف Y وذلك من خلال استخدام معادلات نافير ستوك في النظام القطبي

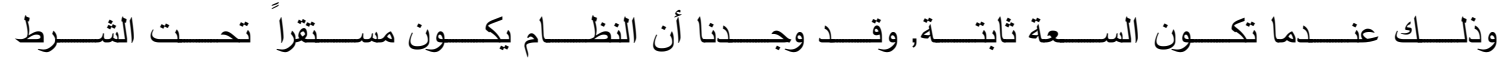
$\cdot \frac{1}{\operatorname{Re}}<\frac{\frac{1}{r}\left(k_{1}^{2} u_{1}+k_{1} k_{2} w_{1}\right)}{\frac{1}{r^{2}}\left(k_{1}^{2}-r^{2} k_{1}^{2} k_{2}^{2}-k_{2}^{2}-r^{2} k_{2}^{4}\right)}$ الكلمات المفتاحية: الشرايين المتضيقة، تدفق الدم ، تحليل الاستقرارية ، المعاملات اللابعدية

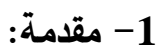

تلعب ظاهرة نقل الدم وحركته في الثرايين دوراً أساسيا في فهم العديد من أمراض الثرايين وهذه الحركة

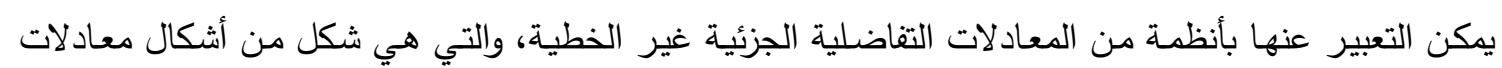

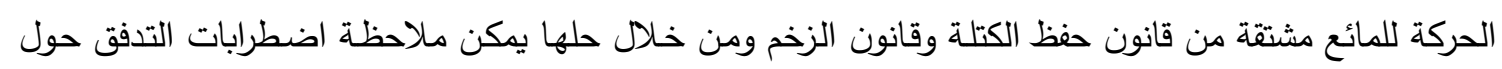


الانحناءات والتقوسات في الثرايين والتي من خلالها يمكن فهم الأضرار الناتجة وكيفية معالجتها وهذا يقودنا إلى

دراسة الاستقرارية والتي تعد من المفاهيم الأساسية في كثير من العلوم وتطبيقاتها.

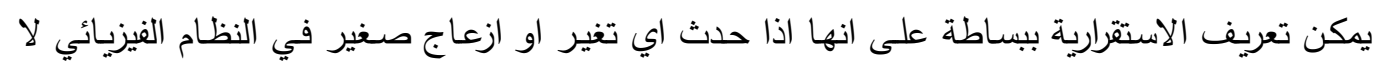

يؤدي في النهاية الى تغير كبير في سلوك النظام رورياضيا معنى الاستقرارية هو تغير بسيط في القيم الابتدائية في

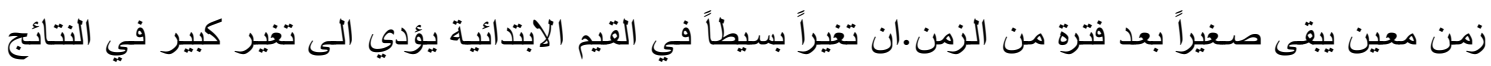

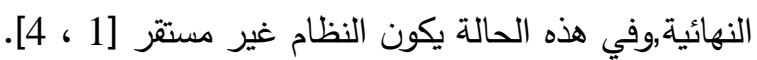

فقد تعلقت دراسة (Cha'o-Kuang Chen and Ming-Chetin) باستقرارية طبقة رقيقة من المائع

يجري في اسطوانة[3].

كما أجرى كل من (Bimal Kumar mishra and 2Nidhi Verma) دراسة على تأثير التضيق على جريان الدم غير النيوتيني في قناة الدم وذلك من خلال ايجاد قوة قص الجدار وايجاد معامل المقاومة[2] وفي عام (2009) عمل كل من (Nidhi Verma and R.S.Parihar) على دراسة تأثير القوة المغناطيسية على فئى

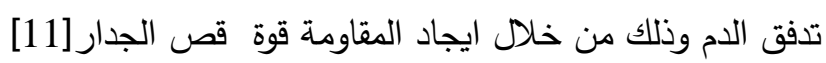
كما قام كل من (S. Sen. and S. Chakravarty) عام 2006 بدراسة تدفق الدم تحت تأثير عملية الانتشار من خلال أنموذج لشريان متضيق ومتفرع، حيث تم استخدام آلية الفروقات المنتهية للحصول على النتائج ولقد لاحظوا ان تأثير التضيق ضئيل على قيم الضغط للجدار الخارجي (Daughter wall)في حين أن تأثير قابلية التمدد للجدار كبيرة[12].

كما عمل كل من (J. C. Misra and G. C. Shit) في عام (2005) على تطوير أنموذج رياضي

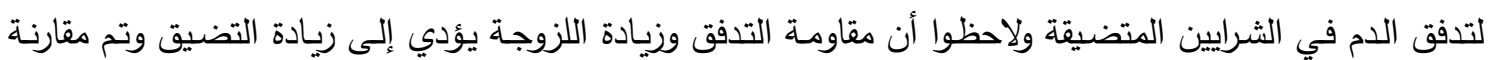
النتائج مع البيانات المتوفرة التي قدمها باحثون آخرون [8]. كذلك عمل (Sapna Ratan Shah and S. U. Siddiqui) في 2011 على حل مشكلة تدفق الدم النيوتونية وغير الخطية في شريان متضيق وذلك باستخدام إحدى الطرائق العددية وبين أن سرعة تدفق الدم وإجهاد

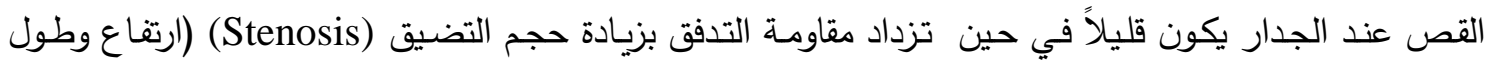
التضيق) [14] ، [15]

كما بين كل من (S. C. Pardhan2 and Sachin Shaw1 and P.V.S. Nmurtty1) تأثير القوة المغناطيسية على جريان الدم وبين ان الزيادة في الدجال المغناطيسي تؤدي إلى قلـة نصف قطر الثريان وسرعة الجريان [13].

2- 2 النموذج والمعادلات الأساسية في الجريان: ليكن لـدينا مقطـع لجريـان الـدم في شـريان متضـيق ومتفرع وهذا التفرع يأخذ شكل الحرف Y كمـا في الشكل(1). 


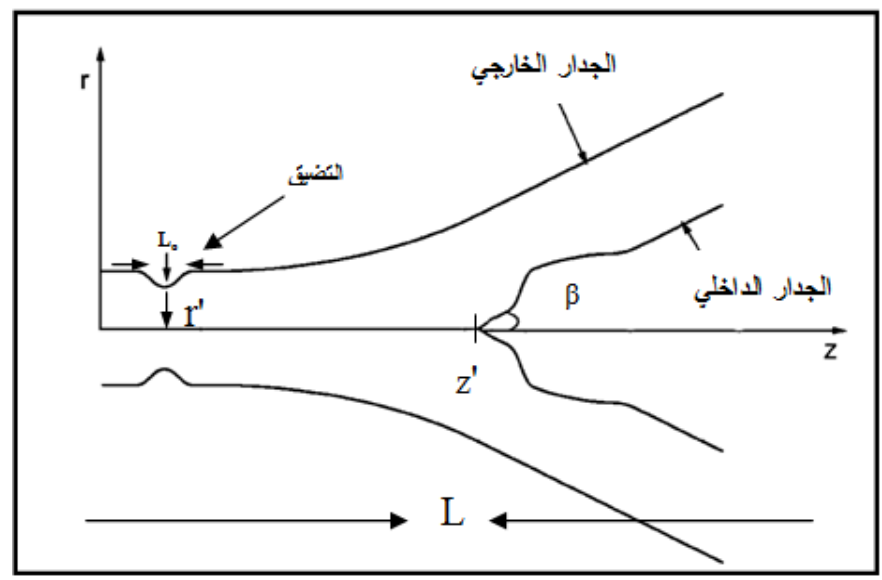

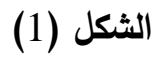

حيث أن:

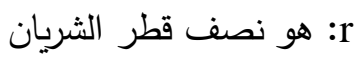
r' L أ هو طول الثربان

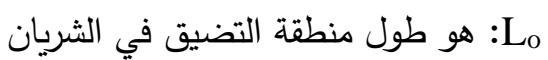
ويمكن تمثيل معادلات جريان الدم هذه بالثكل التالي [12]. $\rho\left(\frac{\partial u^{*}}{\partial t^{*}}+u^{*} \frac{\partial u^{*}}{\partial r^{*}}+w^{*} \frac{\partial u^{*}}{\partial z^{*}}\right)=-\frac{\partial p^{*}}{\partial r^{*}}+\mu\left(\frac{\partial^{2} u^{*}}{\partial r^{2^{*}}}+\frac{1}{r^{*}} \frac{\partial u^{*}}{\partial r^{*}}-\frac{u^{*}}{r^{* 2}}+\frac{\partial^{2} u^{*}}{\partial z^{* 2}}\right)$

$\rho\left(\frac{\partial w^{*}}{\partial t^{*}}+u^{*} \frac{\partial w^{*}}{\partial r^{*}}+w^{*} \frac{\partial w^{*}}{\partial z^{*}}\right)=-\frac{\partial p^{*}}{\partial z^{*}}+\mu\left(\frac{\partial^{2} w^{*}}{\partial r^{* 2}}+\frac{1}{r^{*}} \frac{\partial w^{*}}{\partial r^{*}}+\frac{\partial^{2} w^{*}}{\partial z^{* 2}}\right)$

$\frac{\partial u^{*}}{\partial r^{*}}+\frac{u^{*}}{r^{*}}+\frac{\partial w^{*}}{\partial z^{*}}=0$

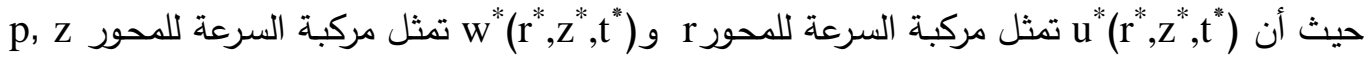

يمثل الضغط , م تمثل الكثافة، $\mu$ تمثل لزوجة الدم

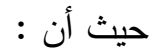

$u^{*}\left(r^{*}, z^{*}, t^{*}\right)=0, \frac{\partial w^{*}\left(r^{*}, z^{*}, t^{*}\right)}{\partial r^{*}}=0 \quad$ on $\quad r^{*}=0 \quad$ for $0 \leq z \leq z$

$u^{*}=\alpha \frac{\partial R_{1}}{\partial t^{*}} \quad, w^{*}\left(r^{*}, z^{*}, t^{*}\right)=0, \quad$ on $\quad r^{*}=R_{1}\left(\mathrm{z}^{*}, \mathrm{t}^{*}\right)$ for all $\mathrm{z}$

$u^{*}=\alpha \frac{\partial R_{2}}{\partial t^{*}} \quad, w^{*}\left(r^{*}, z^{*}, t^{*}\right)=0$, on $\quad r^{*}=R_{2}\left(z^{*}, t^{*}\right) \quad$ for $\quad z \geq z^{\prime}$

حيث أن : n

$\alpha=1 \quad$ for $\quad z \leq z^{\prime} \quad \& \quad \alpha=\sec \beta \quad$ for $\quad z \geq z$

3- المعاملات اللابعدية (Dimensional analysis):

لغرض إيجاد المعادلات اللابعدية للمعادلات (1) و (2) و (3) سوف نفرض بعض القيم اللابعدية[13]. $\mathrm{u}^{*}=\mathrm{uw}_{\mathrm{o}} \quad \mathrm{r}^{*}=\mathrm{rr}_{\mathrm{o}}$ 


$$
\begin{array}{ll}
\mathrm{w}^{*}=\mathrm{ww}_{\mathrm{o}} & \mathrm{z}^{*}=\mathrm{zz}_{\mathrm{o}} \\
\mathrm{p}^{*}=\rho \mathrm{p} w_{o}^{2} & t^{*}=\frac{t r_{o}}{w_{o}}
\end{array}
$$

وبتعويض هذه القيم اللابعدية في المعادلات (1) و (2) و (3) نحصل على معادلات في الحالة اللابعدية.

$\frac{\partial u}{\partial t}+u \frac{\partial u}{\partial r}+w \frac{\partial u}{\partial z}=-\frac{\partial p}{\partial r}+\frac{1}{\operatorname{Re}}\left(\frac{\partial^{2} u}{\partial r^{2}}+\frac{1}{r} \frac{\partial u}{\partial r}-\frac{u}{r^{2}}+\frac{\partial^{2} u}{\partial z^{2}}\right)$

$\frac{\partial w}{\partial t}+u \frac{\partial w}{\partial r}+w \frac{\partial w}{\partial z}=-\frac{\partial p}{\partial z}+\frac{1}{\operatorname{Re}}\left(\frac{\partial^{2} w}{\partial r^{2}}+\frac{1}{r} \frac{\partial w}{\partial r}+\frac{\partial^{2} w}{\partial z^{2}}\right)$

$\frac{\partial u}{\partial r}+\frac{u}{r}+\frac{\partial w}{\partial z}=0$

حيث أن Re هو Reynold's number ومعرف كالآتي:

$\operatorname{Re}=\frac{\rho r_{o} w_{o}}{\mu}$

كذلك فان الثروط في الحالة اللابعدية هي :

$u(r, z, t)=0, \frac{\partial w(r, z, t)}{\partial r}=0 \quad$ on $\quad r=0 \quad$ for $\quad 0 \leq z \leq z$

$u=\alpha \frac{\partial R_{1}}{\partial t}, w(r, z, t)=0, \quad$ on $\quad r=R_{1}(z, t) \quad$ for all $\mathrm{z}$

$u=\alpha \frac{\partial R_{2}}{\partial t}, w(r, z, t)=0, \quad$ on $\quad r=R_{2}(z, t)$ for $z \geq z^{\prime}$

حيث أن:

$\alpha=1 \quad$ for $\quad z \leq z^{\prime} \quad \& \quad \alpha=\sec \beta \quad$ for $\quad z \geq z$

4- 2 - تحليل الاستقرارية:

لغرض تحليل الاستقرارية لأنموذج معادلات جريان الدم المعرف بالمعادلات (7) و (8) و(9) نجزئ كلا

$\left.\begin{array}{l}\mathrm{u}(\mathrm{r}, \mathrm{z}, \mathrm{t})=\mathrm{u}_{1}(\mathrm{r}, \mathrm{z})+\mathrm{u}_{2}(\mathrm{r}, \mathrm{z}, \mathrm{t}) \\ \mathrm{w}(\mathrm{r}, \mathrm{z}, \mathrm{t})=\mathrm{w}_{1}(\mathrm{r}, \mathrm{z})+\mathrm{w}_{2}(\mathrm{r}, \mathrm{z}, \mathrm{t}) \\ \mathrm{p}(\mathrm{r}, \mathrm{z}, \mathrm{t})=\mathrm{p}_{1}(\mathrm{r}, \mathrm{z})+\mathrm{p}_{2}(\mathrm{r}, \mathrm{z}, \mathrm{t})\end{array}\right\}$

من u, w, pباستخدام المعادلات التالية:

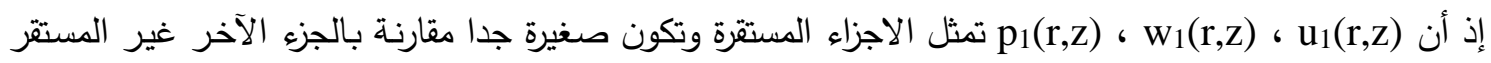

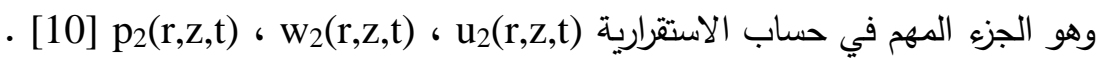

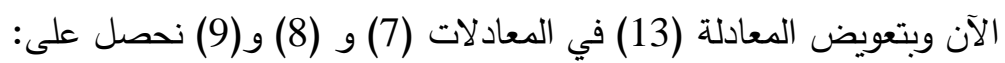

$\frac{\partial\left(u_{1}+u_{2}\right)}{\partial t}+\left(u_{1}+u_{2}\right) \frac{\partial\left(u_{1}+u_{2}\right)}{\partial r}+\left(w_{1}+w_{2}\right) \frac{\partial\left(u_{1}+u_{2}\right)}{\partial z}$

$$
=-\frac{\partial\left(p_{1}+p_{2}\right)}{\partial r}+\frac{1}{\operatorname{Re}}\left(\frac{\partial^{2}\left(u_{1}+u_{2}\right)}{\partial r^{2}}+\frac{1}{r} \frac{\partial\left(u_{1}+u_{2}\right)}{\partial r}-\frac{\left(u_{1}+u_{2}\right)}{r^{2}}+\frac{\partial^{2}\left(u_{1}+u_{2}\right)}{\partial z^{2}}\right) .
$$

$\frac{\partial\left(w_{1}+w_{2}\right)}{\partial t}+\left(u_{1}+u_{2}\right) \frac{\partial\left(w_{1}+w_{2}\right)}{\partial r}+\left(w_{1}+w_{2}\right) \frac{\partial\left(w_{1}+w_{2}\right)}{\partial z}$ 


$$
\begin{gathered}
=-\frac{\partial\left(p_{1}+p_{2}\right)}{\partial z}+\frac{1}{\operatorname{Re}}\left(\frac{\partial^{2}\left(w_{1}+w_{2}\right)}{\partial r^{2}}+\frac{1}{r} \frac{\partial\left(w_{1}+w_{2}\right)}{\partial r}+\frac{\partial^{2}\left(w_{1}+w_{2}\right)}{\partial z^{2}}\right) \\
\frac{\partial\left(u_{1}+u_{2}\right)}{\partial r}+\frac{\left(u_{1}+u_{2}\right)}{r}+\frac{\partial\left(w_{1}+w_{2}\right)}{\partial z}=0
\end{gathered}
$$

وبتبسيط المعادلات (14) و(15) و(16) نحصل على:

$\frac{\partial u_{2}}{\partial t}+u_{1} \frac{\partial u_{1}}{\partial r}+u_{1} \frac{\partial u_{2}}{\partial r}+u_{2} \frac{\partial u_{1}}{\partial r}+u_{2} \frac{\partial u_{2}}{\partial r}+w_{1} \frac{\partial u_{1}}{\partial z}+w_{1} \frac{\partial u_{2}}{\partial z}+w_{2} \frac{\partial u_{1}}{\partial z}+w_{2} \frac{\partial u_{2}}{\partial z}$

$=-\left(\frac{\partial p_{1}}{\partial r}+\frac{\partial p_{2}}{\partial r}\right)+\frac{1}{\operatorname{Re}}\left(\frac{\partial^{2} u_{1}}{\partial r^{2}}+\frac{\partial^{2} u_{2}}{\partial r^{2}}+\frac{1}{r} \frac{\partial u_{1}}{\partial r}+\frac{1}{r} \frac{\partial u_{2}}{\partial r}-\frac{u_{1}}{r^{2}}-\frac{u_{2}}{r^{2}}+\frac{\partial^{2} u_{1}}{\partial z^{2}}+\frac{\partial^{2} u_{2}}{\partial z^{2}}\right)$

$\frac{\partial w_{2}}{\partial t}+u_{1} \frac{\partial w_{1}}{\partial r}+u_{1} \frac{\partial w_{2}}{\partial r}+u_{2} \frac{\partial w_{1}}{\partial r}+u_{2} \frac{\partial w_{2}}{\partial r}+w_{1} \frac{\partial w_{1}}{\partial z}+w_{1} \frac{\partial w_{2}}{\partial z}+w_{2} \frac{\partial w_{1}}{\partial z}+w_{2} \frac{\partial w_{2}}{\partial z}$

$$
=-\left(\frac{\partial p_{1}}{\partial z}+\frac{\partial p_{2}}{\partial z}\right)+\frac{1}{\operatorname{Re}}\left(\frac{\partial^{2} w_{1}}{\partial r^{2}}+\frac{\partial^{2} w_{2}}{\partial r^{2}}+\frac{1}{r} \frac{\partial w_{1}}{\partial r}+\frac{1}{r} \frac{\partial w_{2}}{\partial r}+\frac{\partial^{2} w_{1}}{\partial z^{2}}+\frac{\partial^{2} w_{2}}{\partial z^{2}}\right)
$$

$\frac{\partial u_{1}}{\partial r}+\frac{\partial u_{2}}{\partial r}+\frac{u_{1}}{r}+\frac{u_{2}}{r}+\frac{\partial w_{1}}{\partial z}+\frac{\partial w_{2}}{\partial z}=0$

وبتجزئة المعادلات (17) و (18) و (19) إلى حالتي الاستقرارية والاضطرابات نحصل على:

$u_{1} \frac{\partial u_{1}}{\partial r}+w_{1} \frac{\partial u_{1}}{\partial z}=-\frac{\partial p_{1}}{\partial r}+\frac{1}{\operatorname{Re}}\left(\frac{\partial^{2} u_{1}}{\partial r^{2}}+\frac{1}{r} \frac{\partial u_{1}}{\partial r}-\frac{u_{1}}{r^{2}}+\frac{\partial^{2} u_{1}}{\partial z^{2}}\right)$

$\frac{\partial u_{2}}{\partial t}+u_{1} \frac{\partial u_{2}}{\partial r}+u_{2} \frac{\partial u_{1}}{\partial r}+u_{2} \frac{\partial u_{2}}{\partial r}+w_{1} \frac{\partial u_{2}}{\partial z}+w_{2} \frac{\partial u_{1}}{\partial z}+w_{2} \frac{\partial u_{2}}{\partial z}$

$$
=-\frac{\partial p_{2}}{\partial r}+\frac{1}{\operatorname{Re}}\left(\frac{\partial^{2} u_{2}}{\partial r^{2}}+\frac{1}{r} \frac{\partial u_{2}}{\partial r}-\frac{u_{2}}{r^{2}}+\frac{\partial^{2} u_{2}}{\partial z^{2}}\right)
$$

$u_{1} \frac{\partial w_{1}}{\partial r}+w_{1} \frac{\partial w_{1}}{\partial z}=-\frac{\partial p_{1}}{\partial z}+\frac{1}{\operatorname{Re}}\left(\frac{\partial^{2} w_{1}}{\partial r^{2}}+\frac{1}{r} \frac{\partial w_{1}}{\partial r}+\frac{\partial^{2} w_{1}}{\partial z^{2}}\right)$

$\frac{\partial w_{2}}{\partial t}+u_{1} \frac{\partial w_{2}}{\partial r}+u_{2} \frac{\partial w_{1}}{\partial r}+u_{2} \frac{\partial w_{2}}{\partial r}+w_{1} \frac{\partial w_{2}}{\partial z}+w_{2} \frac{\partial w_{1}}{\partial z}+w_{2} \frac{\partial w_{2}}{\partial z}$

$$
=-\frac{\partial p_{2}}{\partial z}+\frac{1}{\operatorname{Re}}\left(\frac{\partial^{2} w_{2}}{\partial r^{2}}+\frac{1}{r} \frac{\partial w_{2}}{\partial r}+\frac{\partial^{2} w_{2}}{\partial z^{2}}\right)
$$

$\frac{\partial u_{1}}{\partial r}+\frac{u_{1}}{r}+\frac{\partial w_{1}}{\partial z}=0$

$\frac{\partial u_{2}}{\partial r}+\frac{u_{2}}{r}+\frac{\partial w_{2}}{\partial z}=0$

ويمكن فرز المعـادلات (21) و (23) و (25) التي تمثل الحالة غير الزمنيـة (Unsteady State) بكتابتها

بالثكل:

$\frac{\partial u_{2}}{\partial t}+u_{1} \frac{\partial u_{2}}{\partial r}+u_{2} \frac{\partial u_{1}}{\partial r}+u_{2} \frac{\partial u_{2}}{\partial r}+w_{1} \frac{\partial u_{2}}{\partial z}+w_{2} \frac{\partial u_{1}}{\partial z}+w_{2} \frac{\partial u_{2}}{\partial z}$ 


$$
\begin{array}{r}
=-\frac{\partial p_{2}}{\partial r}+\frac{1}{\operatorname{Re}}\left(\frac{\partial^{2} u_{2}}{\partial r^{2}}+\frac{1}{r} \frac{\partial u_{2}}{\partial r}-\frac{u_{2}}{r^{2}}+\frac{\partial^{2} u_{2}}{\partial z^{2}}\right) \\
\frac{\partial w_{2}}{\partial t}+u_{1} \frac{\partial w_{2}}{\partial r}+u_{2} \frac{\partial w_{1}}{\partial r}+u_{2} \frac{\partial w_{2}}{\partial r}+w_{1} \frac{\partial w_{2}}{\partial z}+w_{2} \frac{\partial w_{1}}{\partial z}+w_{2} \frac{\partial w_{1}}{\partial z}+w_{2} \frac{\partial w_{2}}{\partial z} \\
=-\frac{\partial p_{2}}{\partial z}+\frac{1}{\operatorname{Re}}\left(\frac{\partial^{2} w_{2}}{\partial r^{2}}+\frac{1}{r} \frac{\partial w_{2}}{\partial r}+\frac{\partial^{2} w_{2}}{\partial z^{2}}\right)
\end{array}
$$

$\frac{\partial u_{2}}{\partial r}+\frac{u_{2}}{r}+\frac{\partial w_{2}}{\partial z}=0$

الآن نحذف الحدود غير الخطية من المعادلات (26) و(27) و(28) لتحويلها إلى معادلات خطية بالإضافة إلى ذلـك يمكـن حـذف الأنسه

$$
\begin{aligned}
& \text { : نحصل على } \frac{\partial^{2} w_{2}}{\partial r^{2}}<\frac{\partial^{2} w_{2}}{\partial z^{2}} \quad, \frac{\partial^{2} u_{2}}{\partial r^{2}}<<\frac{\partial^{2} u_{2}}{\partial z^{2}} \\
& \frac{\partial u_{2}}{\partial t}+u_{1} \frac{\partial u_{2}}{\partial r}+w_{1} \frac{\partial u_{2}}{\partial z}=-\frac{\partial p_{2}}{\partial r}+\frac{1}{\operatorname{Re}}\left(\frac{1}{r} \frac{\partial u_{2}}{\partial r}-\frac{u_{2}}{r^{2}}+\frac{\partial^{2} u_{2}}{\partial z^{2}}\right) \\
& \frac{\partial w_{2}}{\partial t}+u_{1} \frac{\partial w_{2}}{\partial r}+w_{1} \frac{\partial w_{2}}{\partial z}=-\frac{\partial p_{2}}{\partial z}+\frac{1}{\operatorname{Re}}\left(\frac{1}{r} \frac{\partial w_{2}}{\partial r}+\frac{\partial^{2} w_{2}}{\partial z^{2}}\right) \\
& \frac{\partial u_{2}}{\partial r}+\frac{u_{2}}{r}+\frac{\partial w_{2}}{\partial z}=0
\end{aligned}
$$

5- الاضطراب الحاصل بالاتجاهين r,

لإيجاد الحل لهذه المنظومة من المعادلات، نفرض أن الاضطراب حاصل باتجاهين r و z وان السعة

$\left.\begin{array}{l}u_{2}=A_{1} e^{\alpha t+i\left(k_{1} r+k_{2} z\right)} \\ w_{2}=A_{2} e^{\alpha t+i\left(k_{1} r+k_{2} z\right)} \\ p_{2}=A_{3} e^{\alpha t+i\left(k_{1} r+k_{2} z\right)}\end{array}\right\}$

ثابتة ويمكن أن نفرض الحل بالصورة الآتية [3]:

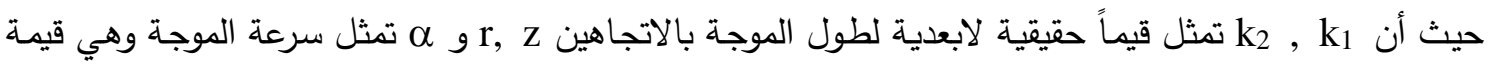
معقدة complex الاضطراب أو تلاشيه على التوالي.

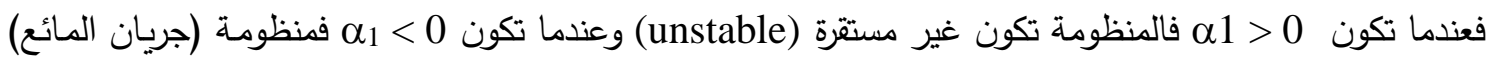
تكون مستقرة (stable) [9].

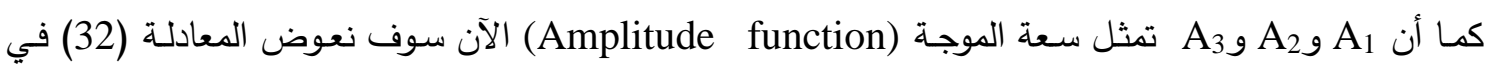

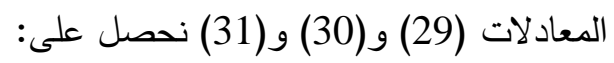

$$
\begin{aligned}
\alpha A_{1} e^{\alpha t+i\left(k_{1} r+k_{2} z\right)} & +u_{1} k_{1} i A_{1} e^{\alpha t+i\left(k_{1} r+k_{2} z\right)}+w_{1} k_{2} i A_{1} e^{\alpha t+i\left(k_{1} r+k_{2} z\right)}=-i k_{1} A_{3} e^{\alpha t+i\left(k_{1} r+k_{2} z\right)} \\
& +\frac{1}{\operatorname{Re}}\left(\frac{1}{r} i k_{1} A_{1} e^{\alpha t+i\left(k_{1} r+k_{2} z\right)}-\frac{1}{r^{2}} A_{1} e^{\alpha t+i\left(k_{1} r+k_{2} z\right)}+\left(i k_{2}\right)^{2} A_{1} e^{\alpha t+i\left(k_{1} r+k_{2} z\right)}\right)
\end{aligned}
$$




$$
\begin{aligned}
& \alpha A_{2} e^{\alpha t+i\left(k_{1} r+k_{2} z\right)}+u_{1} k_{1} i A_{2} e^{\alpha t+i\left(k_{1} r+k_{2} z\right)}+w_{1} k_{2} i A_{2} e^{\alpha t+i\left(k_{1} r+k_{2} z\right)}=-i k_{2} A_{3} e^{\alpha t+i\left(k_{1} r+k_{2} z\right)} \\
& +\frac{1}{\operatorname{Re}}\left(\frac{1}{r} i k_{1} A_{2} e^{\alpha t+i\left(k_{1} r+k_{2} z\right)}+\left(i k_{2}\right)^{2} A_{2} e^{\alpha t+i\left(k_{1} r+k_{2} z\right)}\right) \\
& i k_{1} A_{1} e^{\alpha t+i\left(k_{1} r+k_{2} z\right)}+\frac{1}{r} A_{1} e^{\alpha t+i\left(k_{1} r+k_{2} z\right)}+i k_{2} A_{2} e^{\alpha t+i\left(k_{1} r+k_{2} z\right)}=0 \\
& \text { وبجمع الحدود المتثابهة وترتيب المعادلات (33) و(34) و(35) نحصل على ما يأتي: } \\
& {\left[\left(\alpha+i k_{1} u_{1}+i k_{2} w_{1}-\frac{1}{\operatorname{Re}} \frac{1}{r} i k_{1}+\frac{1}{\operatorname{Re}} \frac{1}{r^{2}}+\frac{k_{2}^{2}}{\operatorname{Re}}\right) A_{1}+i k_{1} A_{3}\right] e^{\alpha t+i\left(k_{1} r+k_{2} z\right)}=0} \\
& {\left[\left(\alpha+i k_{1} u_{1}+i k_{2} w_{1}-\frac{1}{\operatorname{Re}} \frac{1}{r} i k_{1}+\frac{k_{2}^{2}}{\operatorname{Re}}\right) A_{2}+i k_{2} A_{3}\right] e^{\alpha t+i\left(k_{1} r+k_{2} z\right)}=0} \\
& {\left[\left(i k_{1}+\frac{1}{r}\right) A_{1}+i k_{2} A_{2}\right] e^{\alpha t+i\left(k_{1} r+k_{2} z\right)}=0} \\
& \left(\alpha+i k_{1} u_{1}+i k_{2} w_{1}-\frac{1}{\operatorname{Re}} \frac{1}{r} i k_{1}+\frac{1}{\operatorname{Re}} \frac{1}{r^{2}}+\frac{k_{2}^{2}}{\operatorname{Re}}\right) A_{1}+i k_{1} A_{3}=0 \\
& \left(\alpha+i k_{1} u_{1}+i k_{2} w_{1}-\frac{1}{\operatorname{Re}} \frac{1}{r} i k_{1}+\frac{k_{2}^{2}}{\operatorname{Re}}\right) A_{2}+i k_{2} A_{3}=0 \\
& \left(i k_{1}+\frac{1}{r}\right) A_{1}+i k_{2} A_{2}=0
\end{aligned}
$$

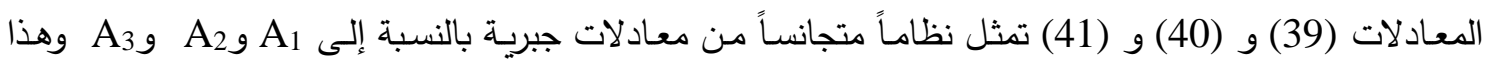

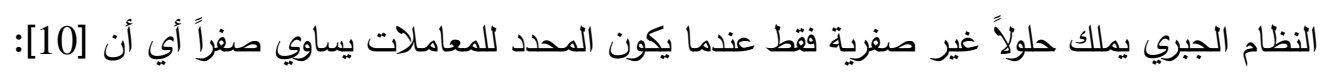

$$
\left|\begin{array}{ccc}
\alpha+i k_{1} u_{1}+i k_{2} w_{1}-\frac{1}{\operatorname{Re}} \frac{1}{r} i k_{1}+\frac{1}{\operatorname{Re}} \frac{1}{r^{2}}+\frac{k_{2}^{2}}{\operatorname{Re}} & 0 & i k_{1} \\
0 & \alpha+i k_{1} u_{1}+i k_{2} w_{1}-\frac{1}{\operatorname{Re}} \frac{1}{r} i k_{1}+\frac{k_{2}^{2}}{\operatorname{Re}} & i k_{2} \\
i k_{1}+\frac{1}{r} & i k_{2} & 0
\end{array}\right|=0
$$

$$
\text { وهذا يؤدي إلى: }
$$$$
-\left[\left(i k_{1}\right)\left(i k_{1}+\frac{1}{r}\right)\left(\alpha+i k_{1} u_{1}+i k_{2} w_{1}-\frac{1}{\operatorname{Re}} \frac{1}{r} i k_{1}+\frac{k_{2}^{2}}{\operatorname{Re}}\right)+\left(i k_{2}\right)^{2}\left(\alpha+i k_{1} u_{1}+i k_{2} w_{1}-\frac{1}{\operatorname{Re}} \frac{1}{r} i k_{1}+\frac{1}{\operatorname{Re}} \frac{1}{r^{2}}+\frac{k_{2}^{2}}{\operatorname{Re}}\right)\right]=0
$$

نقوم بتبسيط المعادلة (42) نحصل على :

$$
\begin{gathered}
-\alpha k_{1}^{2}+i \frac{\alpha k_{1}}{r}-i k_{1}^{3} u_{1}-\frac{k_{1}^{2} u_{1}}{r}-i k_{1}^{2} k_{2} w_{1}-\frac{k_{1} k_{2} w_{1}}{r}+\frac{i k_{1}^{3}}{\operatorname{Re} r}+\frac{k_{1}^{2}}{\operatorname{Re} r^{2}}-\frac{k_{1}^{2} k_{2}^{2}}{\operatorname{Re}}+i \frac{k_{1} k_{2}^{2}}{\operatorname{Re} r} \\
-\alpha k_{2}^{2}-i k_{1} k_{2}^{2} u_{1}-i k_{2}^{3} w_{1}-\frac{i k_{1} k_{2}^{2}}{\operatorname{Re} r}-\frac{k_{2}^{2}}{\operatorname{Re} r^{2}}-\frac{k_{2}^{4}}{\operatorname{Re}}=0
\end{gathered}
$$

وبتجميع وترتيب وتبسيط الحدود للمعادلة (43) نحصل على

$\alpha=\frac{1}{\left(k_{1}^{2}+k_{2}^{2}\right)}\left(-\frac{k_{1}^{2} u_{1}}{r}-\frac{k_{1} k_{2} w_{1}}{r}+\frac{k_{1}^{2}}{\operatorname{Re} r^{2}}-\frac{k_{1}^{2} k_{2}^{2}}{\operatorname{Re}}-\frac{k_{2}^{2}}{\operatorname{Re} r^{2}}-\frac{k_{2}^{4}}{\operatorname{Re}}\right)$ 


$$
\begin{aligned}
& +\frac{i}{\left(k_{1}^{2}+k_{2}^{2}\right)}\left(\frac{\alpha k_{1}}{r}-k_{1}^{3} u-k_{1}^{2} k_{2} w_{1} \frac{k_{1}^{3}}{\operatorname{Re} r}+\frac{k_{1} k_{2}^{2}}{\operatorname{Re} r}-k_{1} k_{2}^{2} u_{1}-k_{2}^{3} w_{1}-\frac{k_{1} k_{2}^{2}}{\operatorname{Re} r}\right) \\
& \alpha=\alpha_{1}+i \alpha_{2} \\
& \alpha_{1}=\frac{1}{\left(k_{1}^{2}+k_{2}^{2}\right)}\left(-\frac{k_{1}^{2} u_{1}}{r}-\frac{k_{1} k_{2} w_{1}}{r}+\frac{k_{1}^{2}}{\operatorname{Re} r^{2}}-\frac{k_{1}^{2} k_{2}^{2}}{\operatorname{Re}}-\frac{k_{2}^{2}}{\operatorname{Re} r^{2}}-\frac{k_{2}^{4}}{\operatorname{Re}}\right) \\
& \text { : } \alpha_{1}>0 \text { - ( عندما تكون } \\
& \frac{1}{\left(k_{1}^{2}+k_{2}^{2}\right)}\left(-\frac{k_{1}^{2} u_{1}}{r}-\frac{k_{1} k_{2} w_{1}}{r}+\frac{k_{1}^{2}}{\operatorname{Re} r^{2}}-\frac{k_{1}^{2} k_{2}^{2}}{\operatorname{Re}}-\frac{k_{2}^{2}}{\operatorname{Re} r^{2}}-\frac{k_{2}^{4}}{\operatorname{Re}}\right)>0 \\
& \frac{1}{\operatorname{Re}}>\frac{\frac{1}{r}\left(k_{1}^{2} u_{1}+k_{1} k_{2} w_{1}\right)}{\frac{1}{r^{2}}\left(k_{1}^{2}-r^{2} k_{1}^{2} k_{2}^{2}-k_{2}^{2}-r^{2} k_{2}^{4}\right)} \\
& \frac{1}{\left(k_{1}^{2}+k_{2}^{2}\right)}\left(-\frac{k_{1}^{2} u_{1}}{r}-\frac{k_{1} k_{2} w_{1}}{r}+\frac{k_{1}^{2}}{\operatorname{Re} r^{2}}-\frac{k_{1}^{2} k_{2}^{2}}{\operatorname{Re}}-\frac{k_{2}^{2}}{\operatorname{Re} r^{2}}-\frac{k_{2}^{4}}{\operatorname{Re}}\right)<0
\end{aligned}
$$

$$
\frac{1}{\operatorname{Re}}<\frac{k_{1}^{2} u_{1}+k_{1} k_{2} w_{1}}{\frac{1}{r}\left(k_{1}^{2}-r^{2} k_{1}^{2} k_{2}^{2}-k_{2}^{2}-r^{2} k_{2}^{4}\right)}
$$

في هذه الحالة يكون النظام مستقراً : $\alpha_{1}=0$ :

$$
\frac{1}{\left(k_{1}^{2}+k_{2}^{2}\right)}\left(-\frac{k_{1}^{2} u_{1}}{r}-\frac{k_{1} k_{2} w_{1}}{r}+\frac{k_{1}^{2}}{\operatorname{Re} r^{2}}-\frac{k_{1}^{2} k_{2}^{2}}{\operatorname{Re}}-\frac{k_{2}^{2}}{\operatorname{Re} r^{2}}-\frac{k_{2}^{4}}{\operatorname{Re}}\right)=0
$$

$$
\frac{1}{\operatorname{Re}}=\frac{\frac{1}{r}\left(k_{1}^{2} u_{1}+k_{1} k_{2} w_{1}\right)}{\frac{1}{r^{2}}\left(k_{1}^{2}-r^{2} k_{1}^{2} k_{2}^{2}-k_{2}^{2}-r^{2} k_{2}^{4}\right)}
$$


$\operatorname{Re}=\frac{\rho r_{o} w_{o}}{\mu}$

$\mu=\rho r_{0} w_{0}\left(\frac{\frac{1}{r}\left(k_{1}^{2} u_{1}+k_{1} k_{2} w_{1}\right)}{\frac{1}{r^{2}}\left(k_{1}^{2}-r^{2} k_{1}^{2} k_{2}^{2}-k_{2}^{2}-r^{2} k_{2}^{4}\right)}\right)$

وهذه الحالة تمثل الحد الفاصل بين الحالة المستقرة وغير المستقرة كما موضح في الثكل (2) :

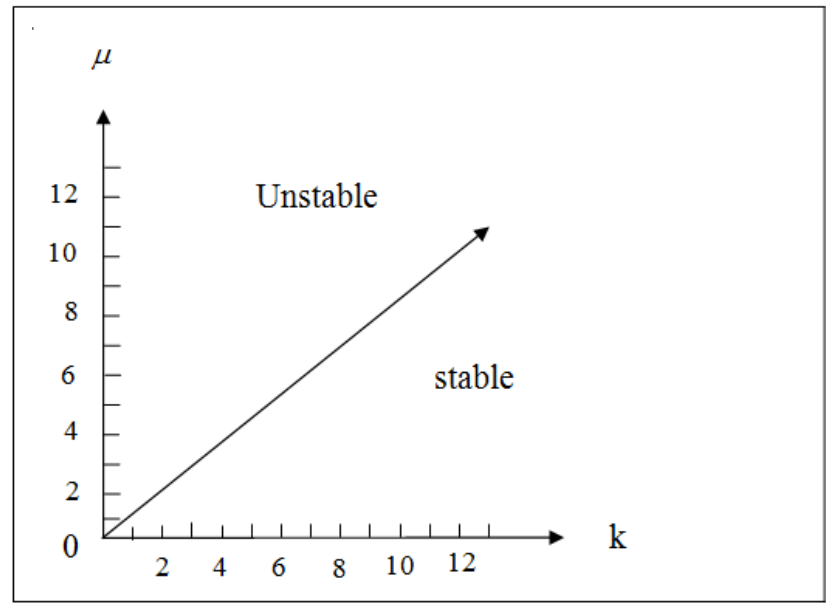

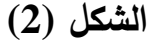

حيث أن :

$k=\rho r_{0} w_{0}\left(\frac{\frac{1}{r}\left(k_{1}^{2} u_{1}+k_{1} k_{2} w_{1}\right)}{\frac{1}{r^{2}}\left(k_{1}^{2}-r^{2} k_{1}^{2} k_{2}^{2}-k_{2}^{2}-r^{2} k_{2}^{4}\right)}\right)$

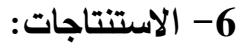

في عملنا هذا قمنا بإيجاد سرعة الموجه م وهي التي تحدد مدى استقرارية الجريان وهي تمثل قيمة معقدة وان القيمـة السـالبة لـ $\alpha_{1}$ تؤثر في حالـة نمو الاضطراب أو تلاشيه. لقد تمـت تجزئـة نظـام

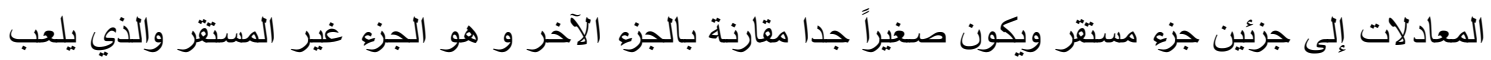
دوراً اساسياً في تحليل الاستقرارية وبعد ايجاد المحدد للنظام استطعنا ان نوجد المعاملات التي تتؤثر على استقرارية جريان الدم في الشريان المذكور حيث ان النظام يكون مستقرا إذا كانت قيمة اللزوجة اقل من قيمة سرعة التدفق والضغط. ان التضيق الحاصل في الشريان يسبب زيادة في جهد القلب لضـخ الدم الى انحاء الجسم وعند زيـادة

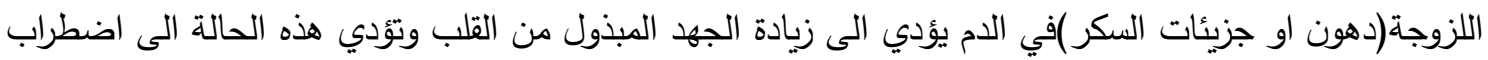
في القلب وهذه هي الحالة غير المستقرة. 


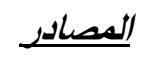

[1] Al-Obaidi, M.F. and Abraham, B.M., (2001): "Stability analysis and chaos in a BendDuct" Raf J. Sci., Vol.12, No. 1, PP 91-99.

[2] Bimal Kumar mishra and 2Nidhi Verma, "Effects of Stenosis on Non-newtonian Flow of Blood in Blood Vessels" , Australian Journal of Basic and Applied Sciences, 4(4): 588- 601, 2010,ISSN 1991-8178, @ 2010, INSInet Publication .

[3] Cha'o-Kuang $\mathrm{Ch}$ en and Ming-Chetin: "Weakly non linear hydrodynamic stability of thin Newtonian fluid flowing on a rotating circular disk", Vol.2009, Article ID 948672, 15 pages.

[4] David L. George (2004): "Numerical Approximation of the Non Linear Shallow Water Equations with Topograph and Dry Bed", PP 5-8.

[5] Fortunato M., Kurizki, G. and Schleich W.P., (1988): "Stabilization of Deterministically chaotic systems by Interference and Quantum Measurement The Ikeda Map case Physical Review Letters", Vol. 80, No.26, PP 530-5733.

[6] Gilbert S. (1980): "Linear Algebra and it's Applications", Second Edition, Academic Press, New York.

[7] Henry M.P and Robert J.S. (1978): "Introduction to Dynamics and Control", McGraw-Hill, London.

[8] J. C. Misra and G. C. Shit, (2005): "Blood flow through arteries in a pathological state: A theoretical Study".

[9] Logan, J. D. (1987): "Applied Mathematics Wiley and Sony".

[10] Mosa, M. F. and Manaa, S.A., "Effects of Radiative heat transfer in the MHD Ekman Layer on aporous plate, Mu'than J. Natural Applied Sci. Series, 7, 268, 1992.

[11] Nidhi Verma and R.S. Parihar "Effects of Magneto - Hydrodynamic and Hematocrit on BloodFlow in an Artery with Multiple Mild Stenosis", International Journal of Applied Mathematics and Computation, Volume 1(1), pp 30-46, 2009.

[12] S. Chakravarty and S. Sen, (2006): "Amathematical model of blood flow and convective diffusion processes in constricted bifurcated arteries", KoreaAustralia Rheology Journal, Vol. 18, No.2, PP 51-65.

[13] Sachi Saw1, P.V.S. Nmurthy1 and S.C. Pardhar2: "The effect of body acceleration on two dimensional flow of casson fluid through artery with asymmetric stenosis", The Open Transport Phenomena, Journal 2010, 2, 55-68.

[14] Sapna Ratan Shah and S.U. Siddiqui: "A comparative study for the NonNewtonian behaviour of blood flow through atherosclerotic arterial segment", Vol. 9, Issue 2, (July-August 2011), Article-019.

[15] Shailes Mishra1, S.U. Siddiqui2 and Amit Medhavi3: "Blood flow through a composite stenosis in an artery with permeable wall", Vol. 6, Issue (June 2011), PP 1798-1813. 